03.4-04 CRYSTAL STRUCTURE OF THIAMIN METHYL ACETYLPHOSPHONATE. By J. Pletcher, A. Turano, W. Furey, M. Sax, Biocrystallography Laboratory, VA Medical Center, Pittsburgh, $\mathrm{Pa}$. 15240, Department of Crysta110graphy, University of Pittsburgh, Pittsburgh, Pa. 15260 and R. Kluger, Department of Chemistry, University of Toronto, Toronto, Ontario.

Thiamin methyl acetylphosphonate is monoclinic, $\mathrm{P}_{2_{1} / \mathrm{c}}$ $a=9.916(8), b=16.840(1), \quad c=15.786(1) \&, \quad B=119.45^{\circ}(4)$, $V=2295 . \AA^{3} \quad Z=4$. The structure was solved using direct methods and refined by full-matrix least squares to an $R$ value of .067 for 1587 observed structure factor amplitudes measured with Mo Ka radiation on a Nonius automatic diffractometer. Various biosynthetic pathways involve thiamin (Vitamin $B_{1}$ ) but there exists a great deal of debate as to conformation and mechanism of action of this coenzyme. Thiamin $C(2)$ adducts are intermediates in the reaction mechanism, many of which are stable under mildly acidic conditions. Substitution occurring on the $\mathrm{C}(2)$ changes the conformation of the molecule with respect to free thiamin. The structure of this inhibitor intermediate was analyzed to investigate whether or not conformational change or retention of configuration is occurring at a specific step in the reaction mechanism. There seem to be many interesting contacts occurring with the phosphonate side chain of this molecule. In particular, one contact from the terminal oxygens seems to have created energetically equal sites causing disorder of one water molecule.

\section{4-05 THE CRYSTAL STRUCTURE OF THIAMINE DINITRATE.}

By D.S.C. Yang, C.S. Yoo, W. Furey, Jr., B.C. Wang, J. Pletcher and M. Sax, Biocrystallography Laboratory, VA Medical Center, Pittsburgh, PA 15240 and the Department of Crystallography, University of Pittsburgh, Pittsburgh, PA 15260.

The structure of the compound was determined by $\mathrm{X}$-ray diffraction. It crystallizes in $P_{T}$ with $z=2$ in a unit cell of dimensions $a=8.93 \AA, b=10.20 \%, c=10.41 \%, \alpha=97.9^{\circ}$, $B=105.1^{\circ}, y=104.4^{\circ}$. It was prepared by titrating thiamine mononitrate with dilute nitric acid. The structure of the mononitrate had been determined by us previously using the $\mathrm{X}$-ray diffraction method also. In addition to reporting the crystal structure of the dinitrate, a detailed comparison with the mononitrate will be presented.
03.4-06 TWO STRUCTURES RELEVANT TO POLYCYCLIC AROMATIC HYDROCARBON CARCINOGENESIS. By J. P. Glusker, H. L. Carrell and D. E. Zacharias, The Institute for Cancer Research, The Fox Chase Cancer Center, 7701 Burholme Avenue, Philadelphia, PA 19111, USA.

The structure of a syn monol expoxide (3,4-epoxy-2methy 1-1,2,3,4-tetrahydro-1-naphthol) has been determined $(a=8.151$ (1) $b=10.007$ (1), $c=6.324$ (1) $\AA$, $\alpha=92.80$ (1) $\beta=104.35$ (1),$\gamma=113.50$ (1) ${ }^{\circ}$, space group (1). The molecule crystallizes with an internal hydrogen bond between the hydroxyl and epoxide oxygen atoms. This forces the conformation of the methy 1 group to be axial. The crystal shows extensive radiation damage during data collection. This structure is a model for the syn diol epoxide of carcinogenic polycyclic aromatic hydrocarbons such as benzo[a]pyrene.

The structure of a covalent adduct of deoxyadenosine with the caxcinogen 7-chloromethyl-12-methylbenz [a] anthracene $\left(\mathbb{N}^{6}-(12-\right.$ methylbenz [a] anthraceny $1-7-m e t h y 1)$ deoxyadenosine, $a=15.815, b=17.965, c=8.871$, space group $2{ }_{1} 2_{1} 2_{1}$ ) has been determined. The molecule lies with the hydrocarbon and sugar groups each perpendicular to the adenine group. The glycosidic torsion angle is $-124^{\circ}$ indicating that the compound exists in this structure in the syn conformation with a hydrogen bond between the hydrogen atom on $0\left(5^{\prime}\right)$ of the ribose and $N(3)$ of adenine (unlike deoxyadenosine which has the anti-conformation in the crystals studied (Acta Cryst. (1969) 19, 111)). The molecules pack with alternate adenine and hydrocarbon groups from different molecules stacking in columns throughout the crystal. The buckled portion of the hydrocarbon group is not involved in the stacking. This research was supported by grants CA10925, CA-22780, CA-06627 and RR-05539 from the National. Institutes of Health, U.S. Public Health Service, a grant BC-242 from the American Cancer Society and by an. appropriation from the Commonwealth of Pennsylvania.

\section{4-07 CRYSTAL STRUCTURE OF A BUILDING UNIT OF} THE FORSSMAN ANTIGEN. By $\mathrm{K}$. Vangehr, P. Luger and H. Paulsen; Institut für Kristallographie der Freien Universität Berlin and Institut für Organische Chemie und Biochemie der Universität Hamburg; West Germany.

The carbohydrate component of the Forssman antigen consists of the pentasaccharide D-GaINAC- $a(1 \rightarrow 3)-D$-GalNAc$\beta-(1 \rightarrow 3)-D-G a 1-\alpha(1-4)-D-G a 1-\beta-(1 \rightarrow 4)-D-G 1 c$ (I) which was recently synthesized (Paulsen,H. \& Bünsch, A. , Angew. Chem. (1980) 92, 929).

We have investigated the crystal structure of 2-acetamido-3-0-(2-acetamido-3, 4, 6-tri-0-acety1-2-deoxy-a-Dgalactopyranosy1) -1, 4, 6-tri-0-acety1-2-deoxy- $\alpha$-D-galacto-pyranose (II) which is a model compound of the terminal disaccharide of (I). Moreover (II) is a building unit of human blood group determinants. The conformation of (II) is of special interest to study interactions with receptor sites in biological activity and may serve as an approach to the structure of the above mentioned biological molecules. Single crystals of (II) are only stable if held in solvent and they are extremely sensitive against mechanical contacts. The structure detemination and refinement was complicated by disorder of two solvent water molecules and of both $\mathrm{N}$ acetyl groups. The result of structure analysis shows that along the glycosidic $\alpha(1 \rightarrow 3)$ linkage atom $C-1$ deviates by $38^{\circ}$ from a trans position to atom $\mathrm{C}-2^{\prime}$ whereas $C-3$, deviates by $23^{\circ}$ from a trans position to $C-2$.

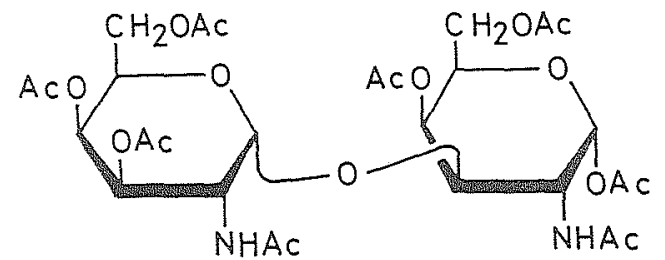

(II) 